\title{
Predictors and prognosis of no-reflow during primary percutaneous coronary intervention
}

Govindan Nair Rajesh, MD, DM, Narayanapillai Jayaprasad, MD, DM (D), Suresh Madhavan, MD, DM, Vasantha Sudha Kumary, MD, DM, Kesavapillai Jayaprakash, MD, DM, Kamarudheenkunju Jameelabeevi Raihanathul Misiriya, MD, DM, Vaikathusseril Lembodaran Jayaprakash, MD, DM, and Raju George, MD, DM

Department of Cardiology, Government Medical College, Kottayam, Kerala, India

\section{ABSTRACT}

The no-reflow phenomenon occurs in a considerable number of patients with ST elevation myocardial infarction (STEMI) undergoing primary reperfusion therapy. Our study aimed to identify clinical, angiographic, and procedural variables that predict this phenomenon in patients with STEMI undergoing primary percutaneous coronary intervention (PPCI), as well as determine the impact of no-reflow on in-hospital mortality. Prospective observational data from 410 patients with STEMI undergoing PPCI were obtained. In this study, diabetes mellitus, late presentation, higher Killip class at admission, anterior wall infarction, proximal site of occlusion, presence of high-grade thrombus, and left ventricular systolic dysfunction at admission were significantly associated with suboptimal coronary flow. Diabetes mellitus, a high thrombus burden, prolonged total ischemic time, and low left ventricular ejection fraction on admission were independent predictors of no-reflow. No-reflow during PPCl was associated with in-hospital mortality more than twice that for normal flow.

KEYWORDS Myocardial infarction; no-reflow; predictors; primary percutaneous intervention

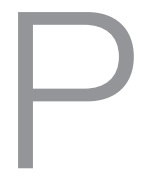

rimary percutaneous coronary intervention (PPCI) has been established as the most effective management strategy to restore antegrade blood flow in ST-elevation myocardial infarction (STEMI). The no-reflow phenomenon occurs in a considerable number of patients with acute STEMI (11\%-41\%) undergoing primary reperfusion therapy. ${ }^{1,2}$ Experimental and clinical studies have shown that the no-reflow phenomenon is associated with large myocardial necrosis and high mortality. ${ }^{3,4}$ Suggested mechanisms for no-reflow or slow flow include coronary microcirculation disturbances, such as distal embolization of thrombus and plaque debris, microvascular damage, and reperfusion injury. ${ }^{1,2}$ A number of clinical, serologic, and angiographic parameters have been shown to be associated with no-reflow. ${ }^{2}$ In addition, a number of treatment strategies have been tried with variable results in no-reflow. ${ }^{5-8}$ Knowing the predictors or risk factors of no-reflow can help prevent this dreaded complication of PPCI. The aim of this study was to identify clinical, angiographic, and procedural factors that predict the no-reflow phenomenon in patients with STEMI undergoing PPCI and to determine the impact of no-reflow on in-hospital mortality.

\section{METHODS}

A total of 455 patients with STEMI underwent PPCI in the Department of Cardiology, Government Medical College, Kottayam, from January 1, 2013, to December 31, 2013. Consecutive patients with acute myocardial infarction who underwent PPCI within 12 hours after appearance of symptoms were included in the study. Exclusion criteria included rescue PCI, patients with STEMI presenting after a $>12$-hour window, patients with coronary dissection (whether spontaneous or procedure related), and patients in whom no stenting was done for various reasons such as unsuitable anatomy or insignificant lesions in coronary angiogram. A total of 410 patients who had undergone PPCI and stenting were included in the study after providing informed consent. STEMI was defined as typical chest pain for more than 30 minutes and either ST segment elevation of $>1 \mathrm{~mm}$ in two consecutive leads or new-onset left bundle

Corresponding author: Narayanapillai Jayaprasad, MD, DM, Department of Cardiology, Government Medical College, Kottayam, Kerala 686008, India (e-mail: jayaprasadn@gmail.com)

Received May 17, 2018; Revised July 30, 2018; Accepted August 6, 2018. 
Table 1. Clinical characteristics of the study patients: no-reflow group vs normal flow group

\begin{tabular}{lccr}
\hline Variable & No-reflow $(n=63)$ & Normal flow $(n=347)$ & $P$ value \\
\hline Age, years (mean \pm SD) & $59.0 \pm 10.9$ & $57.3 \pm 11.4$ & 0.27 \\
Male & $46(73 \%)$ & $273(78.7 \%)$ & 0.99 \\
Previous CAD & $8(12.7 \%)$ & $51(14.7 \%)$ & 0.68 \\
Renal insufficiency & $4(6.3 \%)$ & $14(4 \%)$ & 0.41 \\
Smoking & $27(42.9 \%)$ & $163(47 \%)$ & 0.55 \\
Hypertension & $18(28.6 \%)$ & $91(26.2 \%)$ & 0.70 \\
Diabetes mellitus & $28(44.4 \%)$ & $108(31.1 \%)$ & 0.04 \\
Total ischemic time, $\mathrm{h}$ (mean $\pm \mathrm{SD})$ & $6.63 \pm 2.76$ & $4.50 \pm 2.86$ & $<0.001$ \\
Patients with Killip classes 3 and 4 & $15(23.8 \%)$ & $41(11.8 \%)$ & $<0.001$ \\
Admission ejection fraction & $43.52 \pm 10.5 \%$ & $51.57 \pm 10.6 \%$ & $<0.001$ \\
Proportion of AWMl & $45 / 63(71.4 \%)$ & $150 / 347(43.2 \%)$ & $<0.001$ \\
\hline AWMl indicates anterior wall myocardial infarction; CAD, coronary artery disease. & &
\end{tabular}

branch block. Normal flow was defined as a Thrombolysis in Myocardial Infarction (TIMI) score of 3 after stenting with or without postdilatation. No-reflow was defined as TIMI flow grade $\leq 2$.

Baseline demographic data and data on clinical risk factors were collected. Hypertension was defined as systemic blood pressure $\geq 140 / 90 \mathrm{~mm} \mathrm{Hg}$ or the use of antihypertensive treatment. Diabetes mellitus was defined as fasting blood sugar $\geq 126 \mathrm{mg} / \mathrm{dL}$ or the use of specific treatment. Previous coronary artery disease (CAD) was defined as a history of previous myocardial infarction, acute coronary syndrome, exertional angina, or prior revascularization. Renal insufficiency was defined as a calculated creatinine clearance rate of $<60 \mathrm{~mL} / \mathrm{min}$ as determined by the Cockcroft-Gault equation. Total ischemic time was defined as time from symptom onset to first balloon inflation in minutes. Killip class at presentation and transthoracic echocardiogram ejection fraction at presentation were recorded.

Angiographic data collected included details of the culprit vessel and lesion, such as thrombus burden (low or high), lesion location (proximal, mid, or distal lesion), and TIMI flow grade before the procedure. Thrombus burden was classified as low if the TIMI thrombus class was $\leq 3$ and high if the TIMI thrombus class was $>3$. Patients were divided into normal flow and no-reflow groups according to the coronary flow assessed in coronary angiogram following stenting and postdilatation. Predilatation and postdilatation were done according to operators' discretion. The coronary flow in the infarct-related artery was graded according to the classification used in the TIMI trial. Significant coronary artery stenosis was defined as at least $70 \%$ reduction in the internal diameter of the right coronary artery or left anterior descending or left circumflex coronary artery and their major branches or a $50 \%$ reduction in the internal diameter of the left main coronary artery. Nonsignificant stenosis was defined as coronary arterial narrowing less than significant stenosis. Procedure details were collected, including length, diameter, and type of stent and use of pre- or postdilatation. In-hospital mortality rates of the two groups were compared.

Data were expressed as mean \pm SD. The normal and the no-reflow groups were compared using the chi-square test for discrete variables and unpaired $t$ test for continuous variables according to standard statistical methods. Univariate and multivariate analyses were performed to identify predictors of the no-reflow phenomenon. In all analyses, significance was accepted at $P<0.05$. Statistical analysis was performed with standard statistical software, SPSS Version 20.

\section{RESULTS}

Of the 410 patients included in this study, normal flow was found in 347 patients (84.6\%) and no-reflow in 63 patients $(15.4 \%)$. The mean age was $57.3 \pm 11.4$ years in the normal flow group and $59 \pm 10.9$ years in the no-reflow group. In this study, most patients with normal flow $(42.4 \%)$ were aged 41 to 55 years, whereas most no-reflow patients $(50.8 \%)$ were aged 56 to 70 years, but there was no statistically significant association between advanced age and the incidence of no-reflow. There was no gender difference in occurrence of no-reflow.

Baseline clinical data for the study population are shown in Table 1. The prevalence of diabetes was higher in the noreflow group ( $44.4 \%$ vs $31.1 \%, P=0.04$ ). Total ischemic time was higher in the no-reflow group compared to the normal flow group $(6.63 \pm 2.76$ vs $4.50 \pm 2.86, P<0.001)$. Patients with no-reflow had a higher Killip class than 
Table 2. Angiographic findings and procedural characteristics of the study patients during primary percutaneous coronary intervention

\begin{tabular}{lccc}
\hline Variable & No-reflow $(n=63)$ & Normal flow $(n=347)$ & $P$ value \\
\hline Multivessel CAD & $35(55.6 \%)$ & $157(45.2 \%)$ & 0.13 \\
Proximal vessel lesion & $53(84.1 \%)$ & $250(72 \%)$ & 0.05 \\
Presence of $>$ grade 3 thrombus & $26(41.3 \%)$ & $93(26.8 \%)$ & 0.02 \\
Predilatation & $21(33.3 \%)$ & $91(26.2 \%)$ & 0.24 \\
Initial TIMl flow 0 or 1 & $33(52.4 \%)$ & $162(46.7 \%)$ & 0.41 \\
Stent diameter, mm (mean $\pm \mathrm{SD})$ & $2.99 \pm 0.301$ & $2.99 \pm 0.289$ & 0.64 \\
Stent length, mm (mean $\pm \mathrm{SD})$ & $27.48 \pm 9.3$ & $28.8 \pm 8.9$ & 0.29 \\
Postdilatation & $13(20.6 \%)$ & $43(12.4 \%)$ & 0.08 \\
\hline
\end{tabular}

CAD indicates coronary artery disease; TIMl, Thrombolysis in Myocardial Infarction score.

patients with normal flow: The proportion of patients with Killip classes 3 and 4 was $23.8 \%$ vs $11.8 \%$, respectively. Left ventricular (LV) dysfunction was more frequent in the no-reflow group. The mean LV ejection fraction was $43.52 \pm 10.5$ in the no-reflow group compared with $51.57 \pm 10.6$ in the normal-flow group. Anterior wall myocardial infarction (AWMI) was found to be significantly associated with no-reflow, because $71.4 \%$ of no-reflow group had AWMI, whereas only $43.2 \%$ of the normal-flow group had AWMI.

Angiographic and procedural variables of the two groups are compared in Table 2. The territory of myocardial infarction and the involvement of different culprit vessels were similar among the two groups. Angiographic findings with statistical significance included proximal lesion site in the culprit vessel and thrombus grade. About $84 \%$ of the noreflow group had proximal lesions and $15.9 \%$ had mid or distal lesions. The presence of thrombus grade $>3$ in the culprit vessel was more common in the no-reflow group than in the normal-flow group. Higher than grade 3 thrombus was present in $41.3 \%$ of patients with no-reflow versus $26.8 \%$ of patients with normal flow. The presence of thrombus was significantly associated with the incidence of slow flow. Preprocedure total occlusions (TIMI 0 or 1 flow) were similar in the two groups. Pre- or postdilatation and stent characteristics such as stent length, diameter, and type of stent (bare metal vs drug-eluting stent) were also similar in the two groups.

The only in-hospital outcome measured was in-hospital mortality. No-reflow was significantly associated with high in-hospital mortality. Among the no-reflow group, 10 patients $(7.9 \%)$ died, whereas among the normal-flow group 5 patients $(2.9 \%)$ died ( $P=0.049$, statistically significant).

Multivariate logistic regression analysis was performed to identify the independent predictors of slow flow. The factors that were independently associated with no-reflow were the presence of diabetes (odds ratio $[\mathrm{OR}]=2.222 ; 95 \%$ confidence interval $[\mathrm{CI}], 1.193-4.139 ; P=0.012)$, a high thrombus burden $(\mathrm{OR}=1.875 ; 95 \% \mathrm{CI}, 1.015-3.464$; $P=0$. 045), prolonged total ischemic time $(\mathrm{OR}=2.704$; 95\% CI, 1.437-5.090; $P=0.002)$, and low LV ejection fraction on admission $(\mathrm{OR}=1.060 ; 95 \% \quad \mathrm{CI}$, $1.033-1.088 ; P=0.030)$.

\section{DISCUSSION}

No-reflow is defined as inadequate coronary flow (TIMI grade $\leq 2$ ) despite successful dilatation in the absence of dissection, thrombus, spasm, or high-grade residual stenosis at the original target lesion at the completion of the procedure. The mechanism of no-reflow after PPCI in patients with STEMI is complex. The possible mechanisms of no-reflow include endothelial dysfunction, microvascular disorders, spasm, embolization, and reperfusion injury. In our study, $15.4 \%$ of patients undergoing PPCI developed no-reflow. No-reflow was significantly associated with increased in-hospital mortality (7.9\% compared with $2.9 \%$ among the normal-flow group). In previous studies, no-reflow was not only associated with high in-hospital mortality but also affected long-term prognosis. In the study by Ndrepepa et al, in patients with STEMI treated by PPCI, the no-reflow phenomenon was a strong predictor of 5-year mortality. ${ }^{9}$ In this study involving $>1400$ patients with follow up of $>5$ years, the no-reflow phenomenon after PCI provided prognostic information that was independent of and beyond that provided by infarct size.

In our study, diabetes mellitus, late presentation, higher Killip class at admission, anterior wall infarction, proximal site of occlusion, presence of TIMI grade $>3$ thrombus, and LV systolic dysfunction at admission were significantly associated with suboptimal coronary flow in univariate analysis. Of the variables, diabetes $(\mathrm{OR}=2.222)$, high thrombus burden $(\mathrm{OR}=1.875)$, prolonged total ischemic time $(\mathrm{OR}=2.704)$, and low $\mathrm{LV}$ ejection fraction on admission $(\mathrm{OR}=1.060)$ were independent predictors of no-reflow on multivariate analysis. Hyperglycemia is associated with 
impairment of microvascular function and can cause angiographic slow flow as shown in previous studies. ${ }^{10,11}$ In our study, patients with no-reflow had a longer total ischemic time compared with patients with normal flow. Patients with slow flow also had higher thrombus burden. The presence of grade $>3$ thrombus in the culprit vessel was more frequent in the slow-flow group than in the normal-flow group. Prolonged ischemia leads to edema of distal capillary beds, swelling of myocardial cells, neutrophil plugging, and alterations of capillary integrity. Delayed reperfusion can result in an older, more organized intracoronary thrombus, which may increase the risk of distal embolization during PPCI and increase the chance of no-reflow. ${ }^{12}$ Previous studies have shown that LV ejection fraction $<50 \%$, cardiogenic shock, and tachycardia are independent predictors of final TIMI $\leq 2$ flow. ${ }^{13-15}$ Patients with LV systolic dysfunction resulting from larger infarction can have large microvascular injury, increased LV end-diastolic pressure, and decreased coronary perfusion pressure, leading to suboptimal coronary flow. In our study, stent characteristics like stent length, diameter, and type as well as use of pre- or postdilatation were not predictive of no-reflow. Some studies have shown that long target lesion, initial TIMI flow (0-1), and use of an intra-aortic balloon pump before PCI can independently predict slow flow. ${ }^{16,17}$ Many studies have shown advanced age as an independent risk factor for no-reflow. ${ }^{16,17}$ In our study, there was no significant difference in age between the two groups.

Our study has a few limitations. The only outcome we measured was in-hospital mortality. We did not follow up on patients to determine long-term mortality and adverse cardiac events. We had no data regarding pharmacological treatment during and before the procedure, which might influence achievement of a final TIMI 3 flow. We also had no data regarding frequency of thrombus aspiration and use of intra-aortic balloon pump during PPCI.

Thus, our study showed that no-reflow during PPCI is associated with in-hospital mortality that is more than twice that for normal flow. Factors such as diabetes, prolonged ischemic time, presence of high thrombus burden, and LV systolic dysfunction on admission can predict the occurrence of no-reflow during PPCI. Expecting no-reflow in these patients will help in better management of this dreaded complication.

\section{ORCID}

Narayanapillai Jayaprasad (D) http://orcid.org/0000-0002-1674-339X

1. Harrison RW, Aggarwal A, Ou FS, et al. Incidence and outcomes of no-reflow phenomenon during percutaneous coronary intervention among patients with acute myocardial infarction. Am J Cardiol. 2013; 111(2):178-184. doi:10.1016/j.amjcard.2012.09.015.

2. Schwartz BG, Kloner RA. Coronary no reflow. J Mol Cell Cardiol. 2012;52(4):873-882. doi:10.1016/j.yjmcc.2011.06.009.
3. Reffelmann T, Hale SL, Li G, Kloner RA. Relationship between no reflow and infarct size as influenced by the duration of ischemia and reperfusion. Am J Physiol Heart Circ Physiol. 2002;282(2): H766-H772. doi:10.1152/ajpheart.00767.2001.

4. Ndrepepa G, Tiroch K, Keta D, et al. Predictive factors and impact of no-reflow after primary percutaneous coronary intervention in patients with acute myocardial infarction. Circ Cardiovasc Intervent. 2010;3(1): 27-33. doi:10.1161/CIRCINTERVENTIONS.109.896225.

5. Jaffe R, Dick A, Strauss BH. Prevention and treatment of microvascular obstruction related myocardial injury and coronary no-reflow following percutaneous coronary intervention: a systematic approach. JACC Cardiovasc Interv. 2010;3(7):695-704. doi:10.1016/j.jcin. 2010.05.004.

6. Aung Naing K, Li L, Su Q, Wu T. Adenosine and verapamil for noreflow during primary percutaneous coronary intervention in people with acute myocardial infarction. Cochrane Database Syst Rev. 2013;6: CD009503.

7. Kelbaek H, Terkelsen CJ, Helqvist S, et al. Randomized comparison of distal protection versus conventional treatment in primary percutaneous coronary intervention: the drug elution and distal protection in ST-elevation myocardial infarction (DEDICATION) trial. $J$ Am Coll Cardiol. 2008;51(9):899-905. doi:10.1016/j.jacc.2007.10.047.

8. Stone GW, Webb J, Cox DA, et al. Distal microcirculatory protection during percutaneous coronary intervention in acute ST-segment elevation myocardial infarction: a randomized controlled trial. JAMA. 2005;293(9):1063-1072. doi:10.1001/jama.293.9.1063.

9. Ndrepepa G, Tiroch K, Fusaro M, et al. 5-year prognostic value of no-reflow phenomenon after percutaneous coronary intervention in patients with acute myocardial infarction. J Am Coll Cardiol. 2010; 55(21):2383-2389. doi: 10.1016/j.jacc.2009.12.054.

10. Iwakura $\mathrm{K}$, Ito $\mathrm{H}$, Ikushima $\mathrm{M}$, et al. Association between hyperglycemia and the no-reflow phenomenon in patients with acute myocardial infarction. J Am Coll Cardiol. 2003;41(1):1-7.

11. Capes SE, Hunt D, Malmberg K, et al. Stress hyperglycaemia and increased risk of death after myocardial infarction in patients with and without diabetes: a systematic overview. Lancet 2000;355(9206): 773-778. doi:10.1016/S0140-6736(99)08415-9.

12. Nagata Y, Usuda K, Uchiyama A, et al. Characteristics of the pathological images of coronary artery thrombi according to the infarctrelated coronary artery in acute myocardial infarction. Circ J. 2004; 68(4):308-314.

13. Cura FA, L'Allier PL, Kapadia SR, et al; GUSTO IIb and RAPPORT Investigators. Predictors and prognosis of suboptimal coronary blood flow after primary coronary angioplasty in patients with acute myocardial infarction. Am J Cardiol. 2001;88(2):124-128. doi:10.1016/ S0002-9149(01)01605-8.

14. Mehta RH, Harjai KJ, Cox D, et al. Clinical and angiographic correlates and outcomes of suboptimal coronary flow inpatients with acute myocardial infarction undergoing primary percutaneous coronary intervention. J Am Coll Cardiol. 2003;42(10):1739-1746.

15. Parodi G, Valenti R, Carrabba N, et al. Long-term prognostic implications of nonoptimal primary angioplasty for acute myocardial infarction. Catheter Cardiovasc Interv. 2006;68(1):50-55. doi:10.1002/ ccd.20729.

16. Sabin P, Koshy AG, Gupta PN, et al. Predictors of no-reflow during primary angioplasty for acute myocardial infarction, from Medical College Hospital, Trivandrum. Indian Heart. 2017;69(Suppl 1): S34-S45. doi: 10.1016/j.ihj.2016.12.012.

17. Zhou H, He XY, Zhuang SW, et al. Clinical and procedural predictors of no-reflow in patients with acute myocardial infarction after primary percutaneous coronary intervention. World J Emerg Med. 2014; 5(2):96-102. doi:10.5847/wjem.j.issn.1920-8642.2014.02.003. 